\title{
Uniportal vs. triportal video-assisted thoracic surgery in the treatment of primary pneumothorax - a propensity matched bicentric study
}

\author{
Dania Nachira ${ }^{1 \#}$, Mahmoud Ismail ${ }^{2 \#}$, Elisa Meacci ${ }^{1}$, Edoardo Zanfrini ${ }^{1}$, Amedeo Iaffaldano ${ }^{1}$, Marc \\ Swierzy $^{2}$, Julianna Englisch ${ }^{2}$, Svea Faber ${ }^{1}$, Ramin Raul Ossami Saidy ${ }^{1}$, Maria Letizia Vita ${ }^{1}$, Venanzio \\ Porziella $^{1}$, Jens C. Rueckert ${ }^{2}$, Stefano Margaritora ${ }^{1}$ \\ ${ }^{1}$ Department of General Thoracic Surgery, Fondazione Policlinico Universitario “A. Gemelli”, Rome, Italy; ${ }^{2}$ Competence Center of Thoracic \\ Surgery, Department of Surgery, Charité - Universitätsmedizin Berlin, Berlin, Germany \\ Contributions: (I) Conception and design: D Nachira, M Ismail, E Meacci; (II) Administrative support: S Margaritora, JC Rueckert; (III) Provision of \\ study materials or patients: M Swierzy, ML Vita, V Porziella; (IV) Collection and assembly of data: E Zanfrini, A Iaffaldano, M Swierzy, S Faber, J \\ Englisch, RR Ossami Saidy (V) Data analysis and interpretation: D Nachira, M Ismail, E Meacci; (VI) Manuscript writing: All authors; (VII) Final \\ approval of manuscript: All authors. \\ \#These authors contributed equally to this work. \\ Correspondence to: Dania Nachira, MD. Department of General Thoracic Surgery, Fondazione Policlinico Universitario “A. Gemelli”, Largo A. \\ Gemelli, 8, 00168, Rome, Italy. Email: danynac@libero.it.
}

Background: The role of triportal video-assisted thoracoscopic surgery (VATS) is widely recognized for the treatment of primary spontaneous pneumothorax (PSP). The aim of this study was to assess the effectiveness and the potential advantages of uniportal VATS (U-VATS) for the treatment of PSP compared with triportal VATS.

Methods: A total of 104 triportal $(n=39)$ and uniportal ( $n=65)$ VATS procedures where performed for the treatment of PSP in two University hospitals. The prospectively collected data of postoperative outcomes were retrospectively reviewed and a 1:1 propensity score matching analysis was performed to compare the two VATS approaches.

Results: No major adverse events occurred after operation. Compared with triportal-VATS, UniportalVATS showed the same effectiveness in terms of risk of recurrence (null in both groups), post-operative complications $(\mathrm{P}=1.000)$ and operating time $(66.04 \pm 16.92$ vs. $74.57 \pm 21.38 \mathrm{~min}, \mathrm{P}=0.141)$. However, there was a statistically significant difference in favor of uniportal-VATS in terms of necessity of further access $[0$ vs. 7 (30.4\%), $\mathrm{P}=0.004$ ], chest tube duration (4.39 \pm 1.41 vs. $6.32 \pm 0.94$ days, $\mathrm{P}<<0.001$ ), postoperative hospital stay $(4.78 \pm 1.31$ vs. $6.61 \pm 1.67$ days, $\mathrm{P}<<0.001)$, visual analogue pain score (VAS) at 24 hours $(3.45 \pm 1.41$ vs. $6.44 \pm 2.45, \mathrm{P}<<0.001)$, number of patients who had pain after chest drain removal [1 (4.3\%) vs. $16(69.6 \%)$, $\mathrm{P}<<0.001]$, VAS after drainage removal $(0.11 \pm 0.47$ vs. $2.74 \pm 2.25, \mathrm{P}<<0.001)$, postoperative pain duration $(2.50 \pm 1.20$ vs. $14.82 \pm 37.41$ days, $\mathrm{P}<<0.001)$, pain killers intake $(0.75 \pm 1.06$ vs. $7.53 \pm 3.96$ days, $\mathrm{P}=0.001)$, chronic paresthesia (level scale: 0 to $2 ; 0$ vs. $0.52 \pm 0.66, \mathrm{P}<<0.001$ ), chronic neuralgia (0 vs. $0.43 \pm 0.59$, $\mathrm{P}<<0.001$ ) and cosmetic results (level scale: 0 to $3 ; 2.91 \pm 0.28$ vs. $2.00 \pm 0.77, \mathrm{P}<<0.001$ ).

Conclusions: U-VATS is feasible and safe and may be a less invasive alternative to triportal VATS for the treatment of PSP because of its effectiveness in reducing postoperative pain, paresthesia, hospital stay and in improving cosmetic results.

Keywords: Pneumothorax; triportal video-assisted thoracic surgery (triportal VATS) ; uniportal VATS (U-VATS); pleurectomy

Submitted Feb 16, 2018. Accepted for publication Apr 09, 2018.

doi: 10.21037/jtd.2018.04.124

View this article at: http://dx.doi.org/10.21037/jtd.2018.04.124 


\section{Introduction}

The primary spontaneous pneumothorax (SPS) is a common disorder among young patients, consisting of air accumulation in the pleural space $(1,2)$, because of rupture of pleural blebs or bullae, usually located at the lung apex (Figure 1). Bullectomy and pleurodesis are the most effective treatment which decrease the recurrence rate (3).

The role of video-assisted thoracoscopic surgery (VATS) has been widely recognized to be comparable to thoracotomy in the treatment of spontaneous pneumothorax, with a reduction in the duration of hospital stay, postoperative pain and pulmonary dysfunction (4).

In recent decades, thanks to the development of new endoscopic surgical techniques, there has been an epochal passage where, in addition to surgical effectiveness, more attention has been paid to the degree of invasiveness.

A further significant step forward in terms of less invasiveness was the spread of uniportal VATS (U-VATS).

In fact, U-VATS seems to offer better clinical and economical postoperative outcomes and satisfaction than the standard VATS for the treatment of SPS $(5,6)$.

The aim of this propensity matched bicentric study is to assess the effectiveness and the potential advantages of U-VATS for the treatment of PSP compared to triportal VATS.

\section{Methods}

\section{Patient selections}

The prospective collective data of 98 patients undergone 104 VATS procedures for the treatment of primary PNX, were retrospectively reviewed in this bicentric study. Thirty-four patients underwent triportal VATS approach at "Fondazione Policlinico A. Gemelly" University Hospital, Rome, from February 2012 to May 2016, for a total of
39 procedures (5 patients were operated on both side during this period for bilateral PNX). Thirty-one patients underwent U-VATS approach in the same center, from June 2016 to October 2017.

Thirty-three patients underwent the same U-VATS operation at Charité University Hospital in Berlin, from August 2013 to October 2017, for a total of 34 procedures (1 patient underwent a bilateral operation in two steps).

The indication to the operation was a recurrent PNX or a first episode of PNX with prolonged air-leakage after chest tube placement.

\section{Surgical technique}

All the procedures were performed under general anesthesia and single-lung ventilation.

The patients were positioned in lateral decubitus, with their arms flexed and stretched above their heads.

In all selected cases a pulmonary apicetomy and/or a bullectomy/blebectomy was performed in order to remove the dystrophic and damaged area of the lung parenchyma (Figures 2,3). After resection, a "bubble test" was always conducted to prove the aerostasis. The operation was concluded by selective chemical (talc) pleurodesis of the chest apex or selective mechanical pleurodesis (a parietal pleurectomy from the pleural dome to the 8th-9th rib).

\section{Triportal VATS approach}

The surgeon usually stood on the back-side of the patient, while the assistant held the camera standing opposite to him, on the patient's ventral side.

A $1 \mathrm{~cm}$ incision was performed at VII intercostal space on middle axillary line for the $30^{\circ}$ camera-port. Other two $5 \mathrm{~mm} / 1 \mathrm{~cm}$ incisions were performed on IV intercostal space on the anterior axillary line and on the $\mathrm{IV}-\mathrm{V}$ intercostal space on the posterior mid-clavicular line,
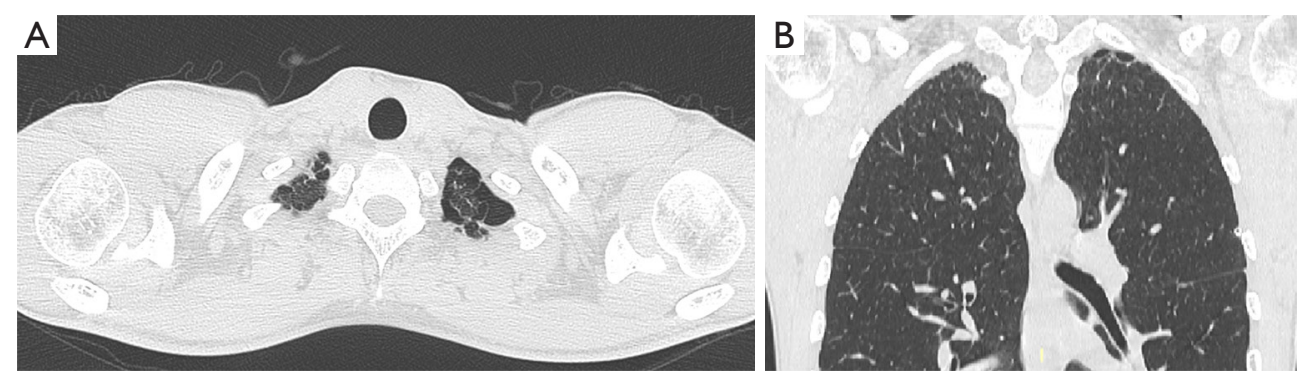

Figure 1 Chest CT scan showing bulla and blebs in the lung apex. 
for the introduction of other two ports for thoracoscopic instruments and stapler.

Intercostal nerve blockade (with Ropivacaine) was performed before the incision and before closing, through the skin from the external side of the chest wall. At the end of the procedure a $24-28$ Fr chest drain was placed through the lower access.

\section{U-VATS approach}

Both surgeons were standing on the ventral side of the

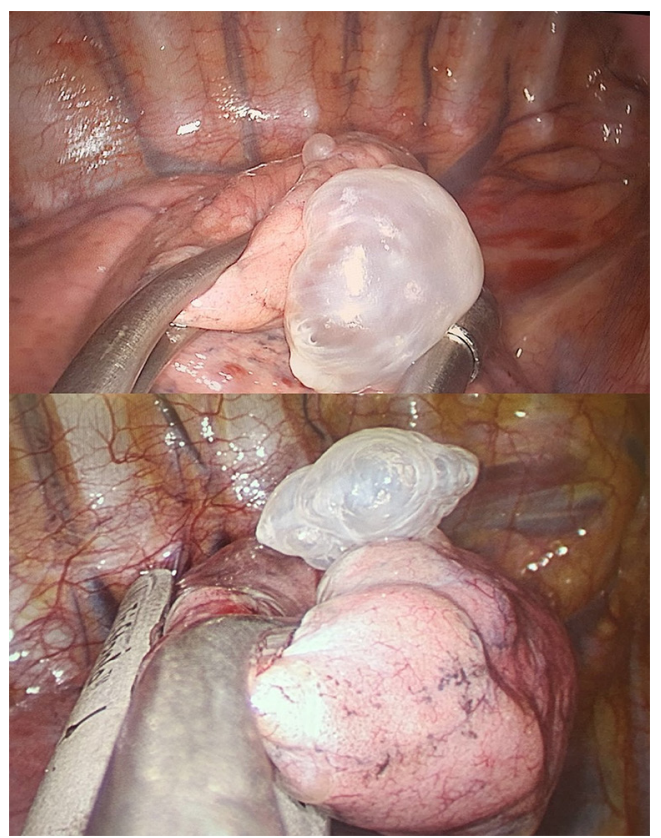

Figure 2 Intraoperative findings and uniportal VATS wedge resection of the dystrophic apex. VATS, video-assisted thoracoscopic surgery. patient, one next to each other, with the assistant at the caudal part, looking at the same screen.

A 2-4 cm incision is made in the IV-V intercostal space on the anterior axillary line. For patients with chest tube, the drainage incision was the uniportal access. A wound protector is always placed, also for preventing the soiling of the $30^{\circ}$ camera that is kept in the upper part of the incision (7). At the end of the operation, the intercostal nerve blockade is performed injecting Ropivacaine from $2^{\text {nd }}$ till $8^{\text {th }}$ intercostal spaces, including this one of the incision, through the parietal pleura under direct visualization of the intercostal nerves. A $24-28$ Fr chest tube is left in place, fixed on the posterior side of the incision (7).

\section{Data collection}

Demographics and clinicopathologic features of patients, like gender, age, smoking habits, comorbidities, previous story of PNX, were prospectively collected. All information regarding operating time, type of parenchymal resection/ blebectomy, type of selective pleurodesis, number of chest drains and postoperative outcomes (in terms of hospital stay, complications, pain, cosmetic results etc.) were also recorded. All patients signed an informed consent before the operation for the treatment and for using their data for scientific analysis.

\section{Propensity score matching and statistical analysis}

To reduce bias resulting from the retrospective nature of this study and to enhance comparability between the two treatment groups, a 1:1 propensity match score analysis was performed using the nearest neighbor matching method with R 3.4.2 software (www.r-project.org).
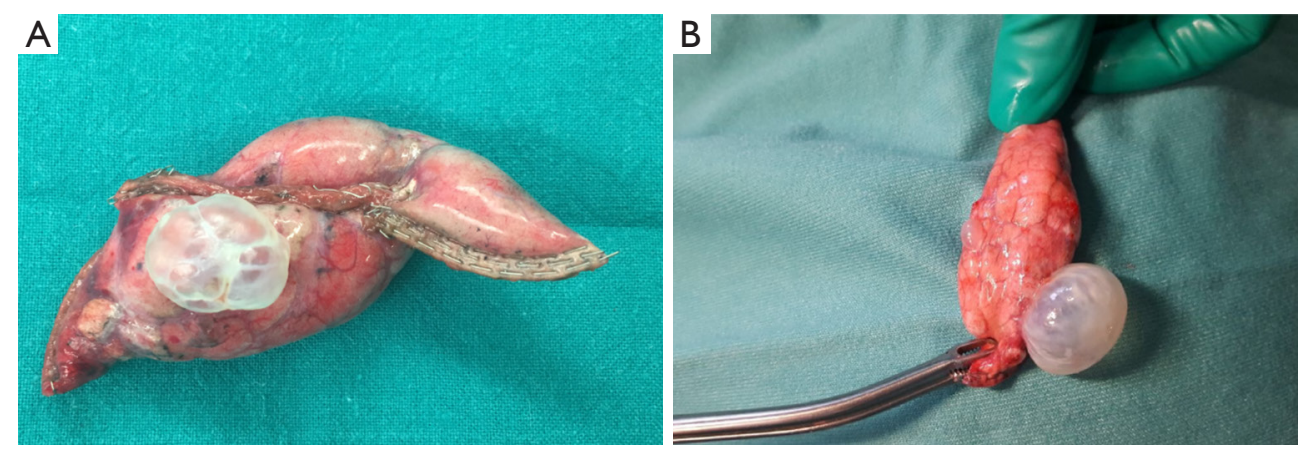

Figure 3 Specimens of bullae. 
Table 1 Clinicopathological characteristics of patients of the entire cohort

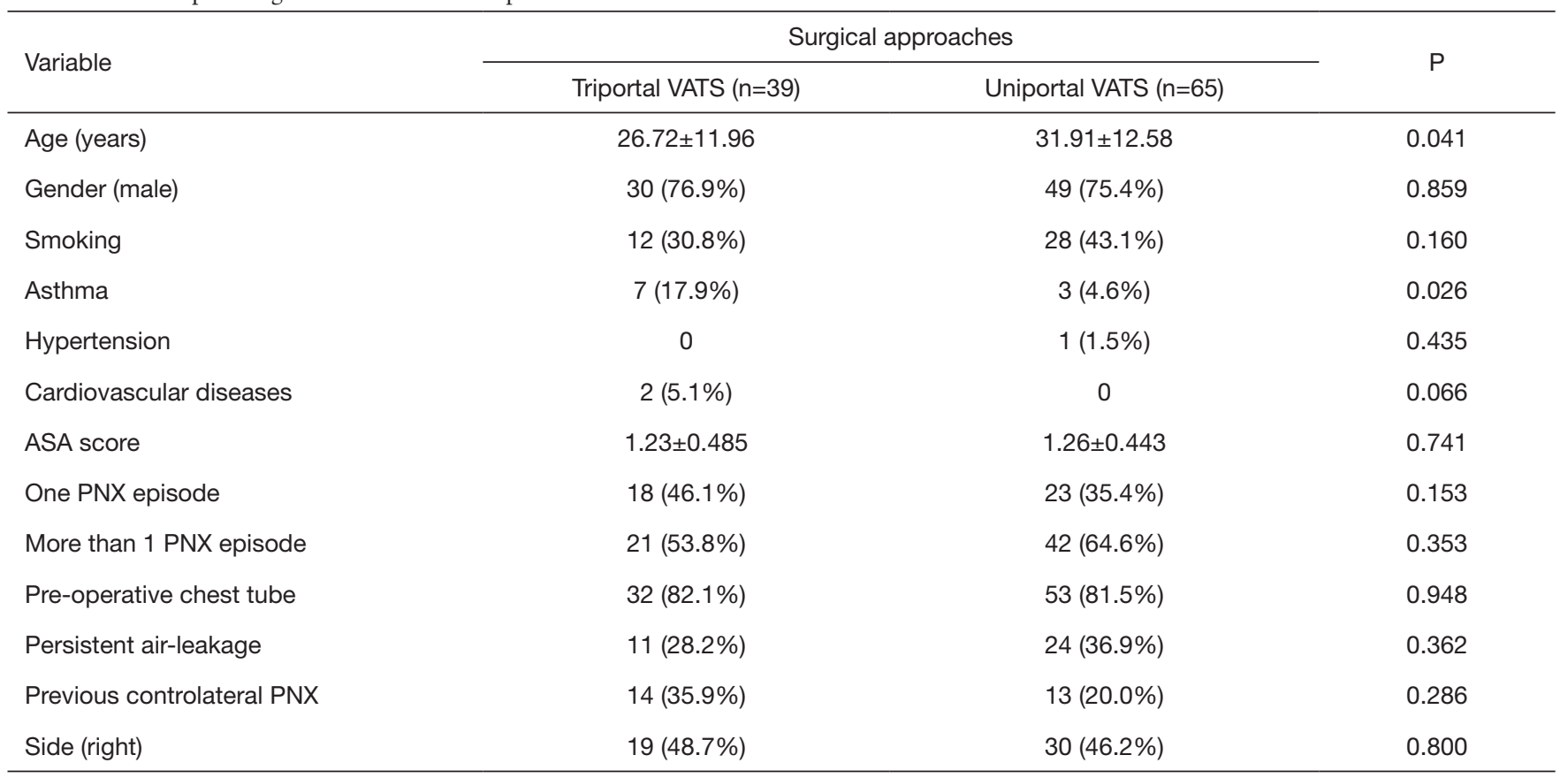

VATS, video-assisted thoracoscopic surgery; ASA, American Society of Anesthesiologists score; PNX, pneumothorax.

Patients were matched by propensity scores based on age, gender, smoking habits, comorbidities, ASA score, side of PNX and number of PNX episodes.

The statistical analysis was performed using SPSS 19.00 software package (Chicago, IL, USA).

Continuous variables were expressed as mean and standard deviation.

Pearson $\chi^{2}$ test and Fischer's exact test were used to compare categorical variables and Student's $t$-test to compare continuous variables.

A $P$ value less than 0.05 was considered statistically significant.

\section{Results}

The preoperative clinicopathological characteristics of the entire cohort of patients treated for PNX either by triportal (39 patients) or uniportal (65 patients) VATS were summarized in Table 1.

The patients in triportal group were younger $(\mathrm{P}=0.041)$ and with a higher incidence of asthma $(\mathrm{P}=0.026)$ than in triportal VATS.

Preoperative comorbidities and baseline characteristics of patients could deeply affect the postoperative outcomes and complications of the two treatments, therefore a 1:1 propensity matched analysis was conducted to reduce the biases in evaluating the best procedure.

After matching, 46 patients (23 couples) were suitable for the analysis.

The distribution of patient characteristics in the pairedcohort, without significant difference, is reported in Table 2.

\section{Effects of VATS approach on postoperative outcomes}

Regarding intraoperative differences between the two approaches, there was no statistical difference in the length of operation time in triportal VATS $v s$. U-VATS $(\mathrm{P}=0.141)$, as well as in the number of patients undergone apicectomies, bullectomies and selective pleurodesis (chemical pleurodesis in all 23 patients in triportal group, while chemical one in 18 and mechanical in 5 in U-VATS) or in the risk of conversion and number of chest drains (Table 3). Nevertheless a higher risk of making a further access for technical difficulties was found in triportal VATS group ( $\mathrm{P}=0.004)$.

Post-operative mortality was null in both group. The mean follow-up was $26.28 \pm 20.58$ months.

Postoperative outcomes and short-term results were also evaluated (Table 4).

There were no reoperation for surgical reasons and 
Table 2 The clinicopathological characteristics of the paired-cohort

\begin{tabular}{|c|c|c|c|}
\hline Variable & \multicolumn{2}{|c|}{ Surgical approaches } & $\mathrm{P}$ \\
\hline Age (years) & $28.74 \pm 13.51$ & $28.70 \pm 12.87$ & 0.991 \\
\hline Gender (male) & $19(82.6 \%)$ & $18(78.3 \%)$ & 0.710 \\
\hline Smoking & $8(34.8 \%)$ & $9(39.1 \%)$ & 0.962 \\
\hline Hypertension & 0 & $1(4.3 \%)$ & 0.312 \\
\hline Cardiovascular diseases & $2(8.7 \%)$ & 0 & 0.148 \\
\hline ASA score & $1.30 \pm 0.559$ & $1.26 \pm 0.449$ & 0.773 \\
\hline One PNX episode & $8(34.8 \%)$ & $6(26.1 \%)$ & 0.522 \\
\hline Persistent air-leakage & $8(34.8 \%)$ & $9(39.1 \%)$ & 0.760 \\
\hline Previous controlateral PNX & $10(43.5 \%)$ & $6(26.1 \%)$ & 0.709 \\
\hline Side (right) & $10(43.5 \%)$ & $13(56.5 \%)$ & 0.376 \\
\hline
\end{tabular}

VATS, video-assisted thoracoscopic surgery; PNX, pneumothorax.

Table 3 The intraoperative results in matched groups

\begin{tabular}{|c|c|c|c|}
\hline Variable & \multicolumn{2}{|c|}{ Surgical approaches } & $\mathrm{P}$ \\
\hline Operation duration (min) & $74.57 \pm 21.38$ & $66.04 \pm 16.92$ & 0.141 \\
\hline Apicetomy & $21(91.3 \%)$ & $22(95.7 \%)$ & 0.550 \\
\hline Other resections (bullectomy) & $3(13.0 \%)$ & $3(13.0 \%)$ & 0.687 \\
\hline Conversion & $1(4.3 \%)$ & 0 & 0.312 \\
\hline Necessity of another access & 7 (30.4\%) & 0 & 0.004 \\
\hline Number of chest drains & $1(4.3 \%)$ & $1(4.3 \%)$ & 1.000 \\
\hline
\end{tabular}

VATS, video-assisted thoracoscopic surgery.

no PNX recurrence in both groups. The incidence of complications was equal (1 postoperative atelectasis in triportal VATS group and 1 pneumonia in uniportal one).

Chest tube duration and postoperative stay were significantly lower after U-VATS surgery $(4.39 \pm 1.41$ vs. $6.32 \pm 0.94$ days and $4.78 \pm 1.31$ vs. $6.61 \pm 1.67$ days, respectively; $\mathrm{P}<<0.001$, Table 4). All variables inherent the evaluation of postoperative pain (level of pain in first postoperative day, after chest drain removal, duration of pain and pain killer intake) were compared and a superiority of U-VATS approach was found, with a significant lower level and less duration of postoperative pain $(\mathrm{P}<<0.001$, Table 4). In particular the incidence and level of postoperative paresthesia and neuralgia 7 days after the operation were null in U-VATS group, while after Triportal VATS 5 patients suffered of both, 5 only of paresthesia, 4 only of neuralgia with a mild-mean level recorded $(0.52 \pm 0.66$ and $0.43 \pm 0.59$ on a $0-2$ scale, respectively), $\mathrm{P}<<0.001$. 
Table 4 The postoperative results in matched groups

\begin{tabular}{|c|c|c|c|}
\hline Variable & \multicolumn{2}{|c|}{ Surgical approaches } & $\mathrm{P}$ \\
\hline Re-operation & 0 & 0 & 1.000 \\
\hline Complications & $1(4.3 \%)$ & $1(4.3 \%)$ & 1.000 \\
\hline PNX recurrence & 0 & 0 & 1.000 \\
\hline Preoperative stay (days) & $3.09 \pm 3.14$ & $2.27 \pm 2.29$ & 0.524 \\
\hline Chest tube duration (days) & $6.32 \pm 0.94$ & $4.39 \pm 1.41$ & $<<0.001$ \\
\hline Pain in 1 postoperative day (VAS scale) & $6.44 \pm 2.45$ & $3.45 \pm 1.41$ & $<<0.001$ \\
\hline Pain after chest drain removal & $16(69.6 \%)$ & $1(4.3 \%)$ & $<<0.001$ \\
\hline Painkillers intake (days) & $7.53 \pm 3.96$ & $0.75 \pm 1.06$ & 0.001 \\
\hline Cosmetic results ( $1-3$ scale) & $2.00 \pm 0.77$ & $2.91 \pm 0.28$ & $<<0.001$ \\
\hline Paresthesia 7 days after $\mathrm{OP}^{*}$ & $0.52 \pm 0.66$ & 0 & $<<0.001$ \\
\hline Neuralgia 7 days after $\mathrm{OP}^{\star}$ & $0.43 \pm 0.59$ & 0 & $<<0.001$ \\
\hline
\end{tabular}

*, level: 0 (null), 1 (medium), 2 (severe). PNX, pneumothorax; VAS, Visual Analogue Score of pain; OP, operation.

Cosmetic results of skin scars were evaluated to be superior by the patients after U-VATS approach $(2.91 \pm 0.28$ vs. $2.00 \pm 0.77$ on a $0-3$ scale, $\mathrm{P}<<0.001)$.

\section{Discussion}

According to last British Society of Thoracic Surgeons (BTS) guidelines of 2010 for the management of PSP, surgical treatment with a bullectomy and a pleurodesis must be advised after the first recurrence of PSP (3).

Open thoracotomy and later VATS have been the main surgical approaches for the treatment of pneumothorax until today. Thoracotomy with pleural abrasion was the original surgical treatment for pneumothorax, described by Tyson and Crandall in 1941 (8), while the first singleincision thoracoscopic surgery (SITS) for bullectomy/ blebectomy was reported by Yamamoto et al. in 1998 (9).

However pleurectomy in Thoracotomy was once used as the gold standard technique as recent as $2003(3,10)$. Indeed the treatment of spontaneous pneumothorax has been developed from open thoracotomy to VATS over recent decades. Because of lower invasiveness, compared with open surgery, conventional VATS has been clearly shown to offer greater advantages in regard to postoperative stay, operative time, chest tube duration and postoperative pain $(11,12)$, improving fast recovery of patients. The single port or single incision or uniportal approach was developed as an even less invasive alternative to the standard multi-port VATS.

In the last years few reports evaluated the efficacy of U-VATS in the treatment of primary PNX; most of them were either retrospective, monocentric and unfocused works that often yielded to conflicting results (13). While some authors reported better postoperative outcomes in terms of operative time, blood loss, postoperative pain (13-16), others didn't (17). Moreover authors experienced limitations in surgical exposure and technical difficulties in performing U-VATS rather than in three port VATS (18).

We conducted this propensity score matching analysis in a bicentric cohort of patients to evaluate and compare U-VATS to three-port VATS for the treatment of PSP, assessing the potential advantages of U-VATS over threeport one. Several parameters, like mortality, recurrence, complications, postoperative stay, preoperative stay, chest tube duration, operative time, pain in I postoperative day, pain after chest drain removal, postoperative pain duration, 
painkiller intake, paresthesia and cosmetic results, were evaluated.

Compared to the three-port VATS group, there was no significant difference in the U-VATS group in terms of mortality, recurrence and complications. The mortality rate and recurrence was $0 \%$ in both groups. Complications were reported in only 1 (4.3\%) patient per each group, no serious complications occurred. We found a significant statistical difference in postoperative stay, operative time, pain in I postoperative day, length of postoperative drainage, pain after chest tube removal, postoperative pain duration, pain killer intake, cosmetic results, paresthesia and neuralgia in favor of U-VATS. In fact U-VATS group was significantly associated with lower paresthesia $(\mathrm{P}<<0.001)$, postoperative pain $(\mathrm{P}<<0.001)$, and higher patient satisfaction in the shortterm.

These good results in terms of pain were similar to other studies (19) and maybe related to the position of the incision in U-VATS (more anterior, where the intercostal space is wider) and the use of a wound protector (instead of one or more trocar), all factors that protect intercostal tissue and nerve-vascular bundle from scratching and compression.

Therefore U-VATS technique was feasible and as clinically safe as the three-port VATS technique and guaranteed excellent outcomes in our series.

In a recent meta-analysis of 2015, Qin et al. (20) drew to similar conclusions: U-VATS did not increase operative time neither prolong postoperative drainage or hospital stay compared with three-port VATS. However, focusing on patients' postoperative pain and paresthesia, U-VATS could reduce them and improve cosmesis and patients' satisfaction.

In the above mentioned meta-analysis (20), only two studies reported a conversion from U-VATS to threeport VATS. Chen et al. (6) stated that the conversion from U-VATS to three-port VATS for hemostasis is really infrequent, contrary to the number of conversions to thoracotomy. Igai et al. (21) reported that only one patient required conversion to a three-port approach because of severe adhesions. In our series there wasn't any conversion or necessity of any further access.

The main limitations of our manuscript are the small sample of patients and the retrospective nature of the study but, to the best of our knowledge, this is the only bicentric study based on a propensity score matching analysis of prospectively collected data.

In conclusion, according to our experience, U-VATS is a safe, feasible and effective technique for the treatment of PSP. And in line with literature evidence, reduction of postoperative paresthesia, neuralgia, pain and length of stay seem to be some of the advantages of U-VATS.

\section{Acknowledgements}

None.

\section{Footnote}

Conflicts of Interest: The authors have no conflicts of interest to declare.

Ethical Statement: This study was approved by the Institutional Review Board of Catholic University of Sacred Hearth (Rome) and Charité - Universitätsmedizin (Berlin).

\section{References}

1. Melton LJ 3rd, Hepper NG, Offord KP. Incidence of spontaneous pneumothorax in Olmsted County, Minnesota: 1950 to 1974. Am Rev Respir Dis 1979;120:1379-82.

2. Swierzy M, Helmig M, Ismail M, et al. Pneumothorax. Zentralbl Chir 2014;139 Suppl 1:S69-86; quiz S87.

3. MacDuff A, Arnold A, Harvey J. Management of spontaneous pneumothorax: British Thoracic Society Pleural Disease Guideline 2010. Thorax 2010;65 Suppl 2:ii18-31.

4. Vohra HA, Adamson L, Weeden DF. Does video-assisted thoracoscopic pleurectomy result in better outcomes than open pleurectomy for primary spontaneous pneumothorax? Interact Cardiovasc Thorac Surg 2008;7:673-7.

5. Salati M, Brunelli A, Xiumè F, et al. Uniportal videoassisted thoracic surgery for primary spontaneous pneumothorax: clinical and economic analysis in comparison to the traditional approach. Interact Cardiovasc Thorac Surg 2008;7:63-6.

6. Chen PR, Chen CK, Lin YS, et al. Single-incision thoracoscopic surgery for primary spontaneous pneumothorax. J Cardiothorac Surg 2011;6:58.

7. Ismail $M$, Swierzy $M$, Nachira D, et al. Uniportal videoassisted thoracic surgery for major lung resections: pitfalls, tips and tricks. J Thorac Dis 2017;9:885-97.

8. Tyson MD, Crandall WB. The surgical treatment of recurrent idiopathic spontaneous pneumothorax. J Thorac Surg1941;10:566-70.

9. Yamamoto H, Okada M, Takada M, et al. Video-assisted 
thoracic surgery through a single skin incision. Arch Surg 1998;133:145-7.

10. Henry M, Arnold T, Harvey J, et al. BTS guidelines for the management of spontaneous pneumothorax. Thorax 2003;58 Suppl 2:ii39-52.

11. Sawada S, Watanabe Y, Moriyama S. Video-assisted thoracoscopic surgery for primary spontaneous pneumothorax: evaluation of indications and long-term outcome compared with conservative treatment and open thoracotomy. Chest 2005;127:2226-30.

12. Goto T, Kadota Y, Mori T, et al. Video-assisted thoracic surgery for pneumothorax: republication of a systematic review and a proposal by the guideline committee of the Japanese association for chest surgery 2014. Gen Thorac Cardiovasc Surg 2015;63:8-13.

13. Huang LZ, Cao QD. Contrast research between single port Video-assisted thoracoscopic surgery and traditional thoracoscope surgery for the treatment of spontaneous pneumothorax. Journal of Ningxia Medical University 2014;36:650-2.

14. Jutley RS, Khalil MW, Rocco G. Uniportal vs. standard three-port VATS technique for spontaneous pneumothorax: comparison of post-operative pain and residual paraesthesia. Eur J Cardiothorac Surg 2005;28:43-6.

15. Kim KS Single-staged uniportal VATS in the supine

Cite this article as: Nachira D, Ismail M, Meacci E, Zanfrini E, Iaffaldano A, Swierzy M, Englisch J, Faber S, Ossami Saidy RR, Vita ML, Porziella V, Rueckert JC, Margaritora S. Uniportal $v s$. triportal video-assisted thoracic surgery in the treatment of primary pneumothorax - a propensity matched bicentric study. J Thorac Dis 2018;10(Suppl 31):S3712-S3719. doi: 10.21037/ jtd.2018.04.124 position for simultaneous bilateral primary spontaneous pneumothorax. J Cardiothorac Surg 2017;12:25.

16. Masmoudi H, Etienne H, Sylvestre R, et al. Three Hundred Fifty-One Patients With Pneumothorax Undergoing Uniportal (Single Port) Video-Assisted Thoracic Surgery. Ann Thorac Surg 2017;104:254-60.

17. Yang HC, Cho S, Jheon S. Single-incision thoracoscopic surgery for primary spontaneous pneumothorax using the SILS port compared with conventional three-port surgery. Surg Endosc 2013;27:139-45.

18. Kim MS, Yang HC, Bae MK, et al. Single-Port Video-Assisted Thoracic Surgery for Secondary SpontaneousPneumothorax: Preliminary Results. Korean J Thorac Cardiovasc Surg 2015;48:387-92.

19. Kang DK, Min HK, Jun HJ, et al. Early outcomes of single-port video-assisted thoracic surgery for primary spontaneous pneumothorax. Korean J Thorac Cardiovasc Surg 2014;47:384-8.

20. Qin SL, Huang JB, Yang YL, et al. Uniportal versus three-port video-assisted thoracoscopic surgery for spontaneous pneumothorax: a meta-analysis. J Thorac Dis 2015;7:2274-87.

21. Igai H, Kamiyoshihara M, Ibe T, et al. Single-incision thoracoscopic surgery for spontaneous pneumothorax using multi-degrees of freedom forceps. Ann Thorac Cardiovasc Surg 2014;20:974-9. 